\title{
Variables predictivas de talla baja adulta en pacientes con hipotiroidismo adquirido grave de origen autoinmune
}

Predictive outcome measures of adult short stature in patients with severe acquired autoimmune hypothyroidism

\author{
Dra. Noelia V. Dujoone ${ }^{a}$ Dra. Natalia A. Gazek ${ }^{a}$ Bioq. Juan M. Lazzatib, \\ Bioq. Mercedes Maceiras ${ }^{b}$, Dra. Alicia Belgorosky y Dra. Viviana C. Herzovich ${ }^{a}$
}

\section{RESUMEN}

El hipotiroidismo por tiroiditis de Hashimoto es la causa más frecuente de disfunción tiroidea en niños.

Nuestro objetivo fue analizar el impacto en la talla final según la talla y el estadio puberal al momento del diagnóstico en menores de 18 años con hipotiroidismo grave de origen autoinmune. De los 79 pacientes, el 78,5\% fueron mujeres. Los que presentaron bocio (el $56 \%$ ) mostraron mejor talla en el diagnóstico que los que no lo tenían (puntaje de desvío estándar de media de talla: 0,2 vs. $-2,42 ; p<0,0001$ ). Cinco niñas (el 6,3\%) presentaron pubertad precoz. De los pacientes con talla final (n: 33), dentro de los que presentaron talla baja al momento del diagnóstico, los púberes tuvieron una talla final significativamente menor que los prepúberes (puntaje de desvío estándar media: $-2,82$ vs. $-1,52 ; \mathrm{p}=0,0311$ ).

Eldiagnóstico tardío dehipotiroidismo graveen pediatría tiene un impacto negativo en la talla final, especialmente, en los pacientes puberales al momento del diagnóstico.

Palabras clave: hipotiroidismo, enfermedad de Hashimoto, estatura, pubertad precoz.

a. Servicio de Endocrinología Infantil.

b. Laboratorio de Endocrinología Infantil.

Hospital de Pediatría Prof. Juan P. Garrahan, Ciudad Autónoma de Buenos Aires.

Correspondencia:

Dra. Noelia V. Dujovne: noedujovne@hotmail. com

Financiamiento:

Ninguno.

Conflicto de intereses: Ninguno que declarar.

Recibido: 21-8-2018 Aceptado: 20-6-2019 http: / / dx.doi.org/10.5546/aap.2019.388

Texto completo en inglés:

http:/ / dx.doi.org/10.5546/ aap.2019.eng.388

Cómo citar: Dujovne NV, Gazek NA, Lazzati JM, Maceiras M, et al. Variables predictivas de talla baja adulta en pacientes con hipotiroidismo adquirido grave de origen autoinmune. Arch Argent Pediatr 2019;117(6):388-391.

\section{INTRODUCCIÓN}

La tiroiditis linfocitaria crónica (TLC) es la causa más frecuente de hipotiroidismo adquirido en la infancia y la adolescencia, con una prevalencia cercana al $2 \%$ en los niños y predominio en el género femenino $(4: 1){ }^{1,2}$ El cuadro clínico es más grave cuanto más tardío es el diagnóstico, con mayor compromiso de la talla final. $^{2}$
Existe escasa bibliografía acerca del impacto en la talla final en los niños con hipotiroidismo adquirido grave (HAG), por lo cual nuestro objetivo fue detectar a aquellos pacientes que presentaban mayor riesgo de baja talla adulta según el estadio puberal y la talla al momento del diagnóstico.

\section{MATERIALES Y MÉTODOS}

Estudio descriptivo retrospectivo de pacientes menores de 18 años con diagnóstico de HAG por TLC, atendidos desde 2008 a 2014 en un centro pediátrico de alta complejidad.

Los criterios de inclusión al momento del diagnóstico fueron los siguientes: hormona estimulante de la tiroides (thyroid stimulating hormone; TSH, por sus siglas en inglés) $>50 \mu \mathrm{IU} / \mathrm{ml}$ con dosajes de hormonas tiroideas bajos para los rangos de referencia según la edad y anticuerpos antitiroideos positivos (anticuerpo antitiroperoxidasa tiroidea -ATPOy/o anticuerpo antitiroglobulina ultrasensible -ATGU). Se excluyeron aquellos pacientes con alguna otra causa que pudiera afectar el crecimiento.

Las variables analizadas fueron género, edad, talla, estadio puberal, edad ósea (EO) al momento del diagnóstico y, en las siguientes visitas, presencia de bocio. La talla se expresó como puntuaciones de desviación estándar (SDE) y se normalizaron para la edad y el género. ${ }^{3} \mathrm{La}$ EO fue evaluada por el mismo endocrinólogo infantil con el método de Greulich y Pyle. En aquellos casos en los que se obtuvieron las tallas de ambos padres, se calcularon las tallas medias parentales. 
Las determinaciones fueron realizadas por quimioluminiscencia, TSH, T3, T4L en plataforma Architect, i4000 Abbott y T4, ATPO y ATGU en plataforma IMMULITE 2000, Siemens. Para TSH y hormonas tiroideas, se utilizaron los valores de referencia previamente publicados. ${ }^{4} \mathrm{El}$ desarrollo puberal fue evaluado según el método de Tanner, y el volumen testicular, utilizando el orquidómetro de Prader. Todos los pacientes, luego del diagnóstico, iniciaron el reemplazo con levotiroxina.

Se consideró talla cercana a la final cuando la velocidad de crecimiento era menor de $0,5 \mathrm{~cm} /$ año y/o presentaron EO adulta. Se definió talla baja cuando era $\leq-2,5 \mathrm{SDE}$ y talla normal, cuando era $>-2,5 \mathrm{SDE}$. Los pacientes con talla cercana a la final fueron divididos en cuatro grupos:

Grupo 1 (G1): prepúber con talla baja.

Grupo 2 (G2): prepúber con talla normal.

Grupo 3 (G3): púber con talla baja.

Grupo 4 (G4): púber con talla normal.

Para analizar las asociaciones entre las distintas variables, se realizó el test de análisis de varianza (analysis of variance; ANOVA, por sus siglas en inglés). Se compararon en cada grupo la talla al momento del diagnóstico y la cercana a la final.

El estudio fue aprobado por el Comité de Ética institucional y se garantizó la protección integral de datos de los pacientes.

\section{RESULTADOS}

Se evaluaron 79 pacientes; el $78 \%$ (n: 62) fueron mujeres. La mediana de edad al momento del diagnóstico fue de 10,9 años, con rango de entre 10 y 12 años.

$\mathrm{Al}$ analizar las asociaciones entre las variables, se encontró que los que presentaron bocio (n: 45) mostraron mejor talla en el diagnóstico que aquellos que no lo presentaban; SDE medio de talla: 0,2 vs. $-2,42(\mathrm{~T}=-5,13 ; \mathrm{p}<0,0001)$.

Solo se obtuvo la talla final de 33 pacientes, ya que, al momento actual, algunos no alcanzaron la talla final y otros perdieron seguimiento (Tabla 1).

Veintitrés pacientes (G2 y G4) se presentaron con talla normal al momento del diagnóstico; 19 de ellos finalizaron con talla normal para la población (8 dentro del rango genético, 2 debajo de este y, en los 9 restantes, no se contó con datos antropométricos del padre). Los 4 restantes finalizaron con talla baja por mala adherencia al tratamiento.

Diez pacientes presentaron talla baja al momento del diagnóstico y los prepuberales (G1) finalizaron con una talla final significativamente mayor que los puberales (G3) $(p=0,0311)$. Los prepúberes tuvieron una mediana de tiempo de seguimiento de 6,83 años, y los púberes, de 2,5 años.

En la Tabla 2, se muestran las características clínicas del G1. Los 4 pacientes finalizaron con talla final normal para la población (SDE: $1,48 \pm 0,37)$. Las niñas presentaron su menarca 1,5 años más tarde que lo descrito en la población normal según Tanner y col.

En la Tabla 3, se muestran las características clínicas del G3. La paciente 2 presentó muy mala adherencia al tratamiento. El paciente 4

Tabla 1. Pacientes con talla final. N: 33

\begin{tabular}{lcccc}
\hline Grupos & N & $\begin{array}{c}\text { Talla al momento } \\
\text { del diagnóstico (SDE) }\end{array}$ & $\begin{array}{c}\text { Talla final } \\
\text { (SDE) }\end{array}$ & $\begin{array}{c}\text { Talla al momento del } \\
\text { diagnóstico/talla final (SDE) }\end{array}$ \\
\hline G 1: Prepúber con talla baja & 4 & $-3 \pm 0,28$ & $-1,52 \pm 0,21$ & $1,48 \pm 0,37$ \\
G 2: Prepúber con talla normal & 5 & $-1,07 \pm 1,27$ & $-1,71 \pm 1,37$ & $-0,64 \pm 1,11$ \\
G 3: Púber con talla baja & 6 & $-3,72 \pm 0,77$ & $-2,82 \pm 1,31$ & $0,9 \pm 0,38$ \\
G 4: Púber con talla normal & 18 & $0,31 \pm 1,09$ & $-0,6 \pm 1,09$ & $-0,91 \pm 0,22$ \\
\hline
\end{tabular}

N: número de pacientes; SDE: puntaje de desvío estándar.

TABLA 2. Evolución de la talla en prepúberes con talla baja al momento del diagnóstico (grupo 1)

\begin{tabular}{lcccccc}
\hline Paciente & Género & $\begin{array}{c}\text { EC } \\
\text { (años) }\end{array}$ & $\begin{array}{c}\text { EC-EO } \\
\text { (años) }\end{array}$ & $\begin{array}{c}\text { Talla al momento } \\
\text { del diagnóstico (SDE) }\end{array}$ & $\begin{array}{c}\text { Menarca } \\
\text { (años) }\end{array}$ & $\begin{array}{c}\text { Talla final } \\
\text { (SDE) }\end{array}$ \\
\hline 1 & $\mathrm{~F}$ & 13,1 & 6,3 & $-2,98$ & 14,6 & $-1,59$ \\
2 & $\mathrm{M}$ & 11,9 & 5,9 & $-3,1$ & & $-1,2$ \\
3 & $\mathrm{~F}$ & 7,25 & 2,25 & $-3,28$ & 13,6 & $-1,67$ \\
4 & $\mathrm{~F}$ & 12,5 & 4,5 & $-2,62$ & 13,9 & $-1,6$ \\
\hline
\end{tabular}

EC: edad cronológica; EO: edad ósea; SDE: puntaje de desvío estándar. 
se diagnosticó a los 15,1 años con pubertad completa. Las pacientes 3 y 6 , por presentar talla baja extrema al momento del diagnóstico, recibieron tratamiento con análogos del factor liberador de gonadotrofinas (gonadotropinreleasing hormone analogue; $a G n R H$, por sus siglas en inglés). La paciente número 3 inició $a G n R H$ a los 10 años con Tanner 3 y talla -3,73 SDE, y suspendió el tratamiento a los 11,8 años con $\mathrm{EO}=12,5$ años. Presentando su menarca a los 13 años, su talla final fue $-3,13$ SDE.

La paciente número 6 alcanzó una talla final normal para la población, pero debajo de su rango genético (-0,76 SDE). Llamativamente, al momento del diagnóstico (13,8 años), ya había presentado su menarca con un atraso de la EO de 6 años y una talla -3,77 SDE. Recibió $a G n R H$ durante 10 meses, que se suspendió por dificultades de provisión, y logró una talla final de -1,9 SDE.

Cinco niñas (el 6,3\%) se presentaron con pubertad precoz (PP) al momento del diagnóstico (Tabla 4).

\section{DISCUSIÓN}

El HAG en los niños produce detención del crecimiento y un profundo retraso en la maduración esquelética, como consecuencia de una osificación y mineralización retrasada, combinada con la atenuación y "down regulación" de la hormona de crecimiento y del factor de crecimiento insuline-like. ${ }^{5,6}$

Una vez iniciado el tratamiento con levotiroxina, los niños experimentan una fase de crecimiento compensador, durante el cual la tasa de maduración esquelética supera la ganancia de estatura, lo que resulta en un déficit de la talla final. Este fenómeno es exacerbado por el inicio de la pubertad central, que, a menudo, ocurre poco después de haber comenzado el reemplazo hormonal. ${ }^{7}$

El objetivo de nuestro trabajo fue describir a la población de pacientes pediátricos con HAG por TLC y analizar el impacto en la talla cercana a la final, según el SDE de la talla y el estadio puberal al momento del diagnóstico, para detectar al grupo de pacientes con mayor riesgo de baja talla adulta.

TABLA 3. Evolución de la talla en púberes con talla baja al momento del diagnóstico (grupo 3)

\begin{tabular}{|c|c|c|c|c|c|c|}
\hline Paciente & Género & $\begin{array}{c}\text { EC } \\
\text { (años) }\end{array}$ & $\begin{array}{l}\text { EC-EO } \\
\text { (años) }\end{array}$ & $\begin{array}{c}\text { Talla al momento } \\
\text { del diagnóstico } \\
\text { (SDE) }\end{array}$ & $\begin{array}{c}\text { Tanner al momento } \\
\text { del diagnóstico }\end{array}$ & $\begin{array}{l}\text { Talla final } \\
\text { (SDE) }\end{array}$ \\
\hline 1 & $\mathrm{~F}$ & 13,4 & 5,4 & $-3,06$ & III & $-2,27$ \\
\hline 2 & $\mathrm{~F}$ & 14,5 & 3,5 & $-4,16$ & IV & $-3,89$ \\
\hline 3 & $\mathrm{~F}$ & 9,3 & 4,3 & $-4,68$ & II & $-3,1$ \\
\hline 4 & M & 15,1 & 7,3 & $-3,13$ & $\mathrm{IV} \mathrm{T}=20 \mathrm{ml}$ & $-2,69$ \\
\hline 5 & M & 14,7 & 6,9 & $-2,9$ & $\mathrm{III} \mathrm{T}=12 \mathrm{ml}$ & $-1,66$ \\
\hline 6 & $\mathrm{~F}$ & 13,9 & 6,1 & $-3,7$ & IV & $-1,9$ \\
\hline
\end{tabular}

EC: edad cronológica; EO: edad ósea; SDE: puntaje de desvío estándar; T: vol. testicular (ml).

TABLA 4. Pacientes con pubertad precoz central

\begin{tabular}{|c|c|c|c|c|c|c|c|}
\hline & Paciente & $\begin{array}{l}\text { EC al momento } \\
\text { del diagnóstico } \\
\text { (años) }\end{array}$ & $\begin{array}{c}\text { Talla al momento } \\
\text { del diagnóstico } \\
\text { (SDE) }\end{array}$ & $\begin{array}{c}\text { Tanner al } \\
\text { momento } \\
\text { del diagnóstico }\end{array}$ & $\begin{array}{l}\text { Menarca } \\
\text { (años) }\end{array}$ & $\begin{array}{l}\text { Talla final } \\
\text { (SDE) }\end{array}$ & $\begin{array}{c}\text { Límite } \\
\text { inferior } \\
\text { de RG } \\
\text { (SDE) }\end{array}$ \\
\hline Antes del & 1 & 11,8 & $-0,16$ & IV & 10 & $-2,55$ & $-3,53$ \\
\hline diagnóstico & 2 & 10,5 & $-0,24$ & IV & 10 & $-1,75$ & $-2,71$ \\
\hline del hipotiroidismo & 3 & 10 & $-0,2$ & IV & 10 & $-2,65$ & $-2,2$ \\
\hline Luego del & 4 & 8,33 & 1,88 & III & 9,4 & $-0,88$ & $-0,89$ \\
\hline $\begin{array}{l}\text { diagnóstico } \\
\text { del hipotiroidismo }\end{array}$ & 5 & 6,25 & 0,43 & I & 9 & $-0,16$ & $-1,48$ \\
\hline
\end{tabular}

EC: edad cronológica; SDE: puntaje de desvío estándar; RG: rango genético 
Las formas de presentación sin bocio tuvieron mayor afectación de la talla. Seguramente, se debió a que su presencia fue un signo clínico que alertó sobre patología tiroidea.

El atraso de la EO al momento del diagnóstico no fue predictor de una mayor tasa de crecimiento, ya que, luego de haber iniciado el reemplazo hormonal, rápidamente, se produjo un avance en la maduración esquelética, que coincidió con lo descrito por Pantsiouou y col. ${ }^{8}$

En nuestro trabajo, al igual que lo descrito por Chiesa y col., ${ }^{9}$ se demostró que un determinante de la talla final no solo fue el déficit de talla al momento del diagnóstico, sino también el tener la pubertad avanzada.

Existen diferentes propuestas de tratamientos coadyuvantes del crecimiento, tales como $a \mathrm{GnRH}$, somatocrinina (growth hormone-releasing hormone; $\mathrm{GHrh}$, por sus siglas en inglés) o inhibidores de la aromatasa (IA). Nebesio y col. compararon la talla final de 13 niños con hipotiroidismo grave (TSH > $150 \mu \mathrm{UI} / \mathrm{ml}), 6$ de los cuales recibieron tratamiento con terapias coadyuvantes (IA, GHrh y / o $a G n R H)$ y 7, solo levotiroxina. No se encontraron diferencias entre ambos grupos. ${ }^{10}$

Se reportaron 6 niños tratados con $a G n R H$, con falla de crecimiento grave, mayor edad cronológica, progresión puberal y avance de la EO, quienes alcanzaron la misma estatura que 17 niños con menor compromiso de talla al momento del diagnóstico tratados solo con levotiroxina. ${ }^{11}$

Reportes de casos aislados ${ }^{12-15}$ demostraron que el tratamiento combinado de GHrh y $a G n R H$, sumado a levotiroxina, lograba una importante ganancia de SDE de talla en los adolescentes con hipotiroidismo grave y mal pronóstico de talla final.

Los trabajos publicados acerca de la eficacia del uso de terapias adyuvantes de crecimiento en pacientes con HAG en la infancia y la adolescencia tienen varias limitaciones, como el bajo número de pacientes, diseños retrospectivos, variables de tiempos de seguimiento, diferentes dosis de inicio de levotiroxina y ajustes subsiguientes, sumados a la falta de estandarización en el uso de terapias adyuvantes.

Si bien la limitante de nuestra serie es el bajo número de pacientes de los subgrupos que alcanzan la talla final, se considera que el diagnóstico tardío del hipotiroidismo grave en pediatría tiene un impacto negativo en esta, especialmente en aquellos púberes con talla baja al momento del diagnóstico, en los cuales se debería considerar el uso de terapias adyuvantes del crecimiento para mejorar su pronóstico de talla.

Estudios aleatorizados, controlados y prospectivos son necesarios para dilucidar los factores modificables, relacionados con la pérdida del potencial de talla en los niños con HAG.

\section{CONCLUSIÓN}

El diagnóstico tardío de hipotiroidismo grave en pediatría tiene un impacto negativo en la talla final, especialmente, en los pacientes puberales al momento del diagnóstico.

\section{REFERENCIAS}

1. Caturegli P, Kimura H, Rocchi R, Rose NR. Autoinmune thyroid diseases. Curr Opin Rheumatol. 2007; 19(1):44-8.

2. De Vries L, Bulvik S, Phillip M. Chronic autoimmune thyroiditis in children and adolescents: at presentation and during long-term follow-up. Arch Dis Child. 2009; 94(1):33-7.

3. Comité Nacional de Crecimiento y Desarrollo. Guía para la evaluación del crecimiento físico. $3{ }^{\text {ra }}$ ed. Buenos Aires: Sociedad Argentina de Pediatría; 2013.

4. Chaler EA, Fiorenzano R, Chilelli C, Llinares V, et al. Age-specific thyroid hormone and thyrotropin reference intervals for a pediatric and adolescent population. Clin Chem Lab Med. 2012; 50(5):885-90.

5. Brent GA. The molecular basis of thyroid hormone action. N Engl J Med. 1994; 311(13):847-53.

6. Robson H, Siebler T, Shalet SM, Williams GR. Interactions between GH, IGF-1, glucocorticoids, and thyroid hormones during skeletal growth. Pediatr Res. 2002; 52(2):137-47.

7. Boersma B, Otten BJ, Stoelinga GB, Wit JM. Catch-up growth after prolonged hypothyroidism. Eur J Pediatr. 1996; 155(5):362-7.

8. Pantsiouou S, Stanhope R, Uruena M, Preece MA, et al. Growth prognosis and growth after menarche in primary hypothyroidism. Arch Dis Child. 1991; 66(7):838-40.

9. Chiesa A, Gruñeiro de Papendieck L, Keselman A, Heinrich JJ, et al. Final height in long-term primary hypothyroid children. J Pediatr Endocrinol Metab. 1998; 11(1):51-8.

10. Nebesio T, Wise M, Perkins S, Eugster E. Does clinical management impactheight potential in childrenwith severe acquired hypothyroidism? J Pediatr Endocrinol Metab. 2011; 24(11-12):893-6.

11. Quintos JB, Salas M. Use of growth hormone and gonadotropin releasing hormone agonist in addition to L-thyroxine to attain normal adult height in two patients with severe Hashimoto's thyroiditis. J Pediatr Endocrinol Metab. 2005; 18(5):515-21.

12. Teng L, Bui H, Bachrach L, Lee P, et al. Catch-up growth in severe juvenile hypothyroidism: treatment with a GnRH analog. J Pediatr Endocrinol Metab. 2004; 17(3):345-54.

13. Minamitani K, Murata A, Ohnishi H, Wataki K, et al. Attainment of normal height in severe juvenile hypothyroidism. Arch Dis Child. 1994; 70(5):429-30.

14. Bruder JM, Samuels MH, Bremer WJ, Ridgway EC, et al. Hypothyroidism-induced macroorchidism: use of a gonadotropin-releasing hormone agonist to understand its mechanism and augment adul stature. J Clin Endocrinol Metab. 1995; 80(1):11-6. 